\title{
ON SOME CASES OF CAUCHY PROBLEM
}

\author{
Silvia-Otilia Corduneanu \\ Department of Mathematics \\ Gh. Asachi Technical University of Iaşi \\ 11 Copou Blvd., RO-6600 Iaşi, Romania \\ silvcord@math.tuiasi.ro
}

\begin{abstract}
The study of almost periodic measures depending on the parameter $t \in \mathbb{R}$ suggested to consider some cases of Cauchy problem. The solutions are functions belonging to $\mathcal{C}^{1}(\mathbb{R}, a p(G))$, where $a p(G)$ is the space of almost periodic measures on a locally compact abelian group $G$. A generalization of these cases is given by replacing $a p(G)$ with a locally convex space.
\end{abstract}

Keywords: Cauchy problem, almost periodic measure, almost periodic function.

\section{Introduction}

Let $X$ be a locally convex space, $Y$ a Banach algebra and $\bullet: Y \times X \rightarrow X$ a bilinear mapping. Consider $y \in Y$ and the linear operator $A: X \rightarrow X$, where $A x=y \bullet x, x \in X$. In our paper we give hypotheses for solving the Cauchy problem

$$
\left\{\begin{array}{l}
\frac{d u(t)}{d t}=A u(t), \quad t \in \mathbb{R} \\
u(0)=u_{0}
\end{array}\right.
$$

where $u_{0} \in X$ and $u \in \mathcal{C}^{1}(\mathbb{R}, X)$. We also give some applications for functions which have values in the space of almost periodic measures $a p(G)$. The set $a p(G)$, of all almost periodic measures on a locally compact abelian group $G$, is a locally convex space with respect to a topology which is called the product topology. If $\nu$ is a bounded measure and $\mu$ is an almost periodic measure, their convolution, $\nu * \mu$, is also an almost periodic measure. These considerations allow us to discuss the Cauchy problem

$$
\left\{\begin{array}{l}
\frac{d u(t)}{d t}=\nu * u(t), \quad t \in \mathbb{R}, \\
u(0)=u_{0} .
\end{array}\right.
$$

Here $u \in \mathcal{C}^{1}(\mathbb{R}, a p(G)), \nu$ is a bounded measure on $G$ and $u_{0} \in a p(G)$.

The original version of this chapter was revised: The copyright line was incorrect. This has been corrected. The Erratum to this chapter is available at DOI: 10.1007/978-0-387-35690-7_44

V. Barbu et al. (eds.), Analysis and Optimization of Differential Systems

(C) IFIP International Federation for Information Processing 2003 


\section{Preliminaries}

Consider a Hausdorff locally compact abelian group $G$ and let $\lambda$ be the Haar measure on $G$. Let us denote by $\mathcal{C}(G)$ the set of all bounded continuous complex-valued functions on $G$, and by $\mathcal{C}_{U}(G)$ the subset of $\mathcal{C}(G)$ containing the uniformly continuous functions. The sets $\mathcal{C}(G)$ and $\mathcal{C}_{U}(G)$ are Banach algebras endowed with the supremum norm. Throughout this paper, $\|\cdot\|_{u}$ denotes the supremum norm on $\mathcal{C}(G)$. For $f \in \mathcal{C}(G)$ and $a \in G$, the translate of $f$ by a is the function $f_{a}(x)=f(x a)$ for all $x \in G$. Denote by $K(G)$ the linear space of all continuous complex-valued functions on $G$, having a compact support. We denote by $m(G)$ the space of complex Radon measures on $G$. That is, the space of all complex linear functionals $\mu$ on $K(G)$ satisfying the following: for each compact subset $A$ of $G$ there exists a positive number $m_{\mu, A}$ such that $|\mu(f)| \leq m_{\mu, A}\|f\|_{u}$ whenever $f \in K(G)$ and the support of $f$ is contained in $A$. We use $m_{F}(G)$ to denote the subspace of $m(G)$ consisting of all bounded measures, i.e. all linear functionals which are continuous with respect to the supremum norm on $K(G)$. The action of a measure $\mu \in m(G)$ on a function $f \in K(G)$ will be denoted either $\mu(f)$ or $\int_{G} f(x) d \mu(x)$. Corresponding to a measure $\mu \in m(G)$, one defines the variation measure $|\mu| \in m(G)$ by $|\mu|(f)=\sup \{|\mu(g)|: g \in K(G),|g| \leq f\}$ for all $f \in K(G), f \geq 0$. For the Borel functions $f, g$ and the measures $\mu, \nu \in m(G)$, we can define their convolutions $f * g, f * \mu, \nu * \mu$, when that is possible. A translationbounded measure is a measure $\mu \in m(G)$ with the property that for every compact set $A \subseteq G, m_{\mu}(A)=\sup _{x \in G}|\mu|(x A)<\infty$ (L. N. Argabright and J. G. Lamadrid [1974]). The linear space of the translation-bounded measures will be denoted by $m_{B}(G)$. We identify an arbitrary measure $\mu \in m_{B}(G)$ with an element of the space $\left[\mathcal{C}_{U}(G)\right]^{K(G)}$ in the following way: $\mu \equiv\{f * \mu\}_{f \in K(G)}$. From this identification we have the inclusion $m_{B}(G) \subset\left[\mathcal{C}_{U}(G)\right]^{K(G)}$. The space $\left[\mathcal{C}_{U}(G)\right]^{K(G)}$ has the product topology defined by the Banach space structure on $\mathcal{C}_{U}(G)$, hence, $m_{B}(G)$ is a locally convex space of measures with the relative topology. A system of seminorms for the product topology on $m_{B}(G)$ is given by the family $\left\{\|\cdot\|_{f}\right\}_{f \in K(G)}$, where, for a function $f \in K(G),\|\mu\|_{f}=\|f * \mu\|_{u}$, for all $\mu \in m_{B}(G)$. Next we give the definition of an almost periodic function (see E. Hewitt and K. A. Ross [1963]).

Definition 2.1 A function $g \in \mathcal{C}(G)$ is called an almost periodic function on $G$, if the family of translates of $g,\left\{g_{a}: a \in G\right\}$ is relatively compact in the sense of uniform convergence on $G$.

The set $A P(G)$ of all almost periodic functions on $G$ is a Banach algebra with respect to the supremum norm, closed to conjugation. Denote 
by $\hat{G}$ the dual of $G$ and by $[\hat{G}]$ the linear space generated by $\hat{G}$ in $\mathcal{C}(G)$. It is easy to see that $[\hat{G}] \subset A P(G)$. The almost periodic measures are introduced and studied by L. N. Argabright and J. G. Lamadrid (J. G. Lamadrid [1973], L. N. Argabright and J. G. Lamadrid [1990]).

Definition 2.2 The measure $\mu \in m_{B}(G)$ is said to be an almost periodic measure, if for every $f \in K(G), f * \mu \in A P(G)$.

The set $a p(G)$ of all almost periodic measures is a locally convex space with respect to the product topology. If $\nu \in m_{F}(G), \mu \in a p(G)$ then $\nu * \mu \in a p(G)$. Also, if $f \in A P(G)$ and $\mu \in a p(G)$, then the measure $f \mu$, defined by $f \mu(g)=\mu(g f), g \in K(G)$ is an almost periodic measure (see J. G. Lamadrid [1973]). It is also proved that there exists a unique linear functional $M: a p(G) \rightarrow C$ such that $M$ is continuous on $a p(G)$, $M(\mu)=M\left(\delta_{x} * \mu\right)$, for all $x \in G, \mu \in a p(G)$ and $M(\lambda)=1$ (see J. G. Lamadrid [1973]). We used the notation $\delta_{x}$ for the the Dirac measure at $x \in G$. If $\mu \in a p(G)$ we define the mean value of $\mu$ as being the above complex number $M(\mu)$.

\section{A Cauchy problem}

The framework of this section has been suggested by some concrete cases of the Cauchy problem, which are presented in the next section. Let $X$ be a locally convex space with a sufficient family of seminorms $\mathcal{P},(Y,\|\cdot\|)$ a commutative Banach algebra with the unit element denoted by $\theta$. We also consider the Banach algebra $\mathcal{B}(Y)$ of bounded linear operators on $Y$ and a bilinear mapping $\bullet: Y \times X \rightarrow X$ such that

$$
\begin{cases}\left(C_{1}\right) & \theta \bullet x=x, \quad \forall x \in X, \\ \left(C_{2}\right) & y \bullet(z \bullet x)=y z \bullet x, \quad \forall y, z \in Y, \quad \forall x \in X, \\ \left(C_{3}\right) & \forall p \in \mathcal{P}, \quad p(y \bullet x) \leq\|y\| p(x), \quad \forall y \in Y, \quad \forall x \in X .\end{cases}
$$

The following proposition contains simple consequences of the properties regarding the series in commutative Banach algebras.

Proposition 3.1 Consider $y \in Y$. Then for every $t \in \mathbb{R}$, the sequence $\theta+t \frac{y}{1 !}+\cdots+t^{n} \frac{y^{n}}{n !}$ is a Cauchy sequence in the Banach space $Y$ and the limit, which is denoted by $e^{t y}$, has the following properties:
(a) $e^{t y}=\theta$ for $t=0$;
(b) $e^{(t+s) y}=e^{t y} e^{s y} \quad$ for $\quad t, s \in \mathbb{R}$;
(c) $\lim _{t \rightarrow 0}\left\|e^{t y}-\theta\right\|=0$;
(d) $\lim _{t \rightarrow 0}\left\|\frac{e^{t y}-\theta}{t}-y\right\|=0$. 
Next we consider $y \in Y$ and we associate to $y$ the bounded linear operator $A \in \mathcal{B}(Y), A z=y z, z \in Y$ and the family of bounded linear operators depending on parameter $t \in \mathbb{R}, T(t)$, where for all $t \in \mathbb{R}$, $T(t) z=e^{t y} z, z \in Y$. Then $T(t), t \in \mathbb{R}$ is a group of bounded linear operators on $Y$ and $A$ is its generator.

Proposition 3.2 Consider the Cauchy problem

$$
\left\{\begin{array}{l}
\frac{d u(t)}{d t}=y u(t), \quad t \in \mathbb{R} \\
u(0)=u_{0}
\end{array}\right.
$$

where $y, u_{0} \in Y$ and $u \in \mathcal{C}^{1}(\mathbb{R}, Y)$ is the unknown function. Then there exists a unique solution and that is $u(t)=e^{t y} u_{0}, t \in \mathbb{R}$.

Proof. We observe that if we consider the function $A: Y \rightarrow Y, A(z)=$ $y z$ we have that $A \in \mathcal{B}(Y)$, therefore, according to the general theory (see J. A. Goldstein [1985]), the solution of (3.3) is $u(t)=T(t) u_{0}, t \in \mathbb{R}$, where $T(t), t \in \mathbb{R}$ is the group associated to $A$. In fact we can represent the solution in the form $u(t)=e^{t y} u_{0}, t \in \mathbb{R}$.

A function $u \in \mathcal{C}^{1}(\mathbb{R}, X)$ is called solution of the Cauchy problem

$$
\left\{\begin{array}{l}
\frac{d u(t)}{d t}=y \bullet u(t), \quad t \in \mathbb{R}, \\
u(0)=u_{0}
\end{array}\right.
$$

if $u$ satisfies the differential equation and the initial condition, which form this problem. The derivative $\frac{d u}{d t}(t)$ of the function $u: \mathbb{R} \rightarrow X$ is defined taking the limit of differences quotient in the topology of the locally convex space $X$. We say that $u \in \mathcal{C}^{1}(\mathbb{R}, X)$ if there exists the derivative $\frac{d u}{d t}: \mathbb{R} \rightarrow X$ and this is a continuous function.

Lemma 3.1 Consider the functions $u \in \mathcal{C}^{1}(\mathbb{R}, Y), v \in \mathcal{C}^{1}(\mathbb{R}, X)$. Then we have the formula

$$
\frac{d}{d t}[u(t) \bullet v(t)]=u^{\prime}(t) \bullet v(t)+u(t) \bullet v^{\prime}(t), \quad t \in \mathbb{R} .
$$

Proof. Let $p \in \mathcal{P}$ and $t \in \mathbb{R}$. Using $\left(C_{3}\right)$ it follows that for all $h \in \mathbb{R}$, $h \neq 0$ we have 


$$
\begin{aligned}
& p\left[\frac{u(t+h) \bullet v(t+h)-u(t) \bullet v(t)}{h}-u^{\prime}(t) \bullet v(t)-u(t) \bullet v^{\prime}(t)\right] \\
& \leq\left\|\frac{u(t+h)-u(t)}{h}-u^{\prime}(t)\right\| p[v(t+h)] \\
& +\|u(t)\| p\left[\frac{v(t+h)-v(t)}{h}-v^{\prime}(t)\right]+\left\|u^{\prime}(t)\right\| p[v(t+h)-v(t)] .
\end{aligned}
$$

Therefore we obtain

$$
\lim _{h \rightarrow 0} p\left[\frac{u(t+h) \bullet v(t+h)-u(t) \bullet v(t)}{h}-u^{\prime}(t) \bullet v(t)-u(t) \bullet v^{\prime}(t)\right]=0 .
$$

Consequently, formula (3.5) has been proved.

Theorem 3.1 Let $y \in Y$ and consider the family of linear operators on $X, T(t), t \in \mathbb{R}$, where for every $t \in \mathbb{R}, T(t) x=e^{t y} \bullet x, \quad x \in X$. Then for all $x \in X$ we have that

$$
\frac{d}{d t} T(t) x=y \bullet T(t) x, \quad t \in \mathbb{R} .
$$

Proof. Let $p$ be in $\mathcal{P}, t \in \mathbb{R}$ and $x \in X$. For all $h \in \mathbb{R}, h \neq 0$ we have

$$
\begin{gathered}
p\left(\frac{T(t+h) x-T(t) x}{h}-y \bullet T(t) x\right)=p\left(\frac{e^{(t+h) y} \bullet x-e^{t y} \bullet x}{h}-y e^{t y} \bullet x\right) \\
\leq\left\|\frac{e^{(t+h) y}-e^{t y}}{h}-y e^{t y}\right\| p(x) \leq\left\|e^{t y}\right\|\left\|\frac{e^{h y}-\theta}{h}-y\right\| p(x) .
\end{gathered}
$$

Therefore

$$
\lim _{h \rightarrow 0} p\left(\frac{T(t+h) x-T(t) x}{h}-y \bullet T(t) x\right)=0, \text { for all } p \in \mathcal{P}
$$

and this shows (3.6).

Corollary 3.1 Consider $y \in Y, u_{0} \in X$ and the Cauchy problem

$$
\left\{\begin{array}{l}
\frac{d u(t)}{d t}=y \bullet u(t), \quad t \in \mathbb{R} \\
u(0)=u_{0}
\end{array}\right.
$$

where $u \in \mathcal{C}^{1}(\mathbb{R}, X)$ is the unknown function. Then (3.7) has a unique solution $u \in \mathcal{C}^{1}(\mathbb{R}, X)$ and $u(t)=e^{t y} \bullet u_{0}, t \in \mathbb{R}$.

Proof. From Theorem 3.1 it follows that the function $u(t)=e^{y t} \bullet u_{0}$, $t \in \mathbb{R}$ verifies the differential equation contained in (3.7) and from Proposition 3.1 it results that this function satisfies the initial condition. Consider $t>0$. We now prove that if $u \in \mathcal{C}^{1}(\mathbb{R}, X)$ is a solution of (3.7) 
then the function $s \in[0, t] \rightarrow e^{(t-s) y} \bullet u(s) \in X$ has the property that

$$
\frac{d}{d s}\left[e^{(t-s) y} \bullet u(s)\right]=0_{X}, \quad s \in[0, t] .
$$

From Lemma 3.1 and Proposition 3.1 it results that

$$
\frac{d}{d s}\left[e^{(t-s) y} \bullet u(s)\right]=-y e^{(t-s) y} \bullet u(s)+e^{(t-s) y} \bullet u^{\prime}(s), \quad s \in[0, t] .
$$

Combining (3.7) and (3.9) we obtain (3.8). Taking into account that $\mathcal{P}$ is a sufficient family of seminorms on $X$ it results that function $s \in[0, t] \rightarrow e^{(t-s) y} \bullet u(s) \in X$ is constant. The equality $u(0)=u(t)$ gives us that $u(t)=e^{t y} \bullet u_{0}$, and from the fact that $t>0$ is arbitrary we obtain $u(t)=e^{t y} \bullet u_{0}, t \in[0, \infty)$. Finally, similar arguments for $t<0$ lead us to the conclusion that $u(t)=e^{t y} \bullet u_{0}, t \in \mathbb{R}$.

\section{Particular cases of Cauchy problem}

In this section we present some particular cases of the Cauchy problem (3.7). In the first case $Y$ is the Banach algebra $m_{F}(G)$ of bounded measures on a locally compact abelian group $G$. We remind that $m_{F}(G)$ is a commutative Banach algebra with respect the usual norm of bounded measures which is denoted by $\|\cdot\|$. The third operation of $m_{F}(G)$ is the convolution of bounded measures and the unit element is the Dirac measure $\delta_{e} \in m_{F}(G)$, e being the unit element of the group $G$. On the other hand $X$ is the locally convex space $\left(a p(G),\left\{\|\cdot\|_{f}\right\}_{f \in K(G)}\right)$ of all almost periodic measures on $G$. The bilinear mapping $\bullet: Y \times X \rightarrow X$ is given by the convolution between a bounded measure and an almost periodic measure which is an almost periodic measure. We start by giving the following lemma which will play an imporant role in proving Corollary 4.1 .

Lemma 4.1 If $\nu \in m_{F}(G), \mu \in a p(G)$ and $f \in K(G)$ we have that

$$
\|\nu * \mu\|_{f} \leq\|\mu\|_{f}\|\nu\| .
$$

Proof. We notice that

$$
\|\nu * \mu\|_{f}=\|f * \nu * \mu\|_{u}=\sup _{x \in G}\left|\int_{G} f * \mu\left(x y^{-1}\right) d \nu(y)\right| .
$$

Consider $x \in G$. Well-known properties of the integral yield

$$
\left|\int_{G} f * \mu\left(x y^{-1}\right) d \nu(y)\right| \leq \int_{G}|f * \mu|\left(x y^{-1}\right) d|\nu|(y) \leq\|f * \mu\|_{u}\|\nu\|=\|\mu\|_{f}\|\nu\| .
$$


Hence $\|\nu * \mu\|_{f} \leq\|\mu\|_{f}\|\nu\|$.

Corollary 4.1 Consider $\nu \in m_{F}(G), u_{0} \in a p(G)$ and the Cauchy problem

$$
\left\{\begin{array}{l}
\frac{d u(t)}{d t}=\nu * u(t), \quad t \in \mathbb{R}, \\
u(0)=u_{0},
\end{array}\right.
$$

where $u \in \mathcal{C}^{1}(\mathbb{R}, a p(G))$ is an unknown function. Then (4.10) has a unique solution and this is $u \in \mathcal{C}^{1}(\mathbb{R}, a p(G)), u(t)=e^{t \nu} * u_{0}, t \in \mathbb{R}$.

Proof. The properties of the convolution between a bounded measure and an almost periodic measure and Lemma 4.1 enable us to see that the bilinear mapping $*: m_{F}(G) \times a p(G) \rightarrow a p(G)$, satisfies conditions $\left(C_{1}\right),\left(C_{2}\right),\left(C_{3}\right)$ from the preceding section. So, the Cauchy problem (4.10) is a particular case of (3.7). In what follows we can apply Corollary 3.1 and we find the conclusion.

Remark 4.1 In the previous corollary we can replace the locally convex space $a p(G)$ by the Banach space $A P(G)$ and we obtain, in the same manner, a similar result. So, if $\nu \in m_{F}(G), u_{0} \in A P(G)$, then the Cauchy problem

$$
\left\{\begin{array}{l}
\frac{d u(t)}{d t}=\nu * u(t), \quad t \in \mathbb{R} \\
u(0)=u_{0}
\end{array}\right.
$$

has a unique solution and this is $u \in \mathcal{C}^{1}(\mathbb{R}, A P(G)), u(t)=e^{t \nu} * u_{0}, t \in \mathbb{R}$.

In the second case $Y$ is the commutative Banach algebra $A P(G)$ of all almost periodic functions on $G$ and $X$ is the locally convex space of all almost periodic measures on $G,\left(a p(G),\{\|\cdot\|\}_{f \in K(G)}\right)$. The unit element of the $A P(G)$ is the constant function denoted by 1 , which takes the value 1 for all $x \in G$. This time, the bilinear mapping associates to an almost periodic function $g \in A P(G)$ and an almost periodic measure $\mu \in a p(G)$ the almost periodic measure having the density $g$ and the base $\mu$.

Lemma 4.2 If $g \in A P(G), \mu \in a p(G)$ and $f \in K(G)$ we have that

$$
\|g \mu\|_{f} \leq\|g\|_{u}\||f| *|\mu|\|_{u} .
$$

Proof. We have that $\|g \mu\|_{f}=\|f * g \mu\|_{u}=\sup _{x \in G}\left|\int_{G} f\left(x y^{-1}\right) g(y) d \mu(y)\right|$.

Consider $x \in G$. Clearly, the following inequalities hold

$$
\begin{aligned}
& \left|\int_{G} f\left(x y^{-1}\right) g(y) d \mu(y)\right| \leq \int_{G}\left|f\left(x y^{-1}\right) \| g(y)\right| d|\mu|(y) \\
& \leq\|g\|_{u} \int_{G}\left|f\left(x y^{-1}\right)\right| d|\mu|(y) \leq\|g\|_{u}\||f| *|\mu|\|_{u} .
\end{aligned}
$$


Therefore $\|g \mu\|_{f} \leq\|g\|_{u}\||f| *|\mu|\|_{u}$.

Corollary 4.2 Consider $g \in A P(G), u_{0} \in a p(G)$ and the Cauchy problem

$$
\left\{\begin{array}{l}
\frac{d u(t)}{d t}=g u(t), \quad t \in \mathbb{R} \\
u(0)=u_{0}
\end{array}\right.
$$

where $u \in \mathcal{C}^{1}(\mathbb{R}, a p(G))$ is an unknown function. Then (4.12) has a unique solution $u \in \mathcal{C}^{1}(\mathbb{R}, a p(G))$ and $u(t)=e^{t g} u_{0}, t \in \mathbb{R}$.

Proof. We consider the bilinear mapping which associates to an almost periodic function $g \in A P(G)$ and an almost periodic measure $\mu \in a p(G)$ the almost periodic measure having the density $g$ and the base $\mu$. It is easy to see that this bilinear mapping verifies $\left(C_{1}\right),\left(C_{2}\right)$ but does not satisfy $\left(C_{3}\right)$. However, there exists a unique solution and this is $u \in$ $\mathcal{C}^{1}(\mathbb{R}, a p(G)), u(t)=e^{t g} u_{0}, t \in \mathbb{R}$. First let us see that, by Proposition 3.1 and Lemma 4.2 it results that for all $f \in K(G), t \in \mathbb{R}, h \neq 0$ we have

$$
\left\|\frac{e^{(t+h) g} u_{0}-e^{t g} u_{0}}{h}-g e^{t g} u_{0}\right\|_{f} \leq\left\|e^{t g}\right\|_{u}\left\|\frac{e^{h g}-1}{h}-g\right\|_{u}\left\||f| *\left|u_{0}\right|\right\|_{u} .
$$

Hence, for all $t \in \mathbb{R}$ and $f \in K(G)$ we obtain that

$$
\lim _{h \rightarrow 0}\left\|\frac{e^{(t+h) g} u_{0}-e^{t g} u_{0}}{h}-g e^{t g} u_{0}\right\|_{f}=0
$$

and this means that $u(t)=e^{t g} u_{0}, t \in \mathbb{R}$ verifies the differential equation contained in (4.12). By virtue of the properties of the variation measure and taking into consideration Lemma 4.2 we can establish that for a fixed $t>0$ we have

$$
\frac{d}{d s}\left[e^{(t-s) g} u(s)\right]=0_{a p(G)}, \quad s \in[0, t] .
$$

Here, we have denoted by $0_{a p(G)}$, the null measure on $G$. Thus, similar arguments as in the proof of Corollary 3.1 lead us to the conclusion $u(t)=e^{t g} u_{0}, t \in \mathbb{R}$ is the unique solution of (4.12).

\section{Examples and applications}

To illustrate the technique of finding the solution for the Cauchy problems (4.10) and (4.11), we consider some examples. The solution of our first example is a function $u \in \mathcal{C}^{1}(\mathbb{R}, A P(\mathbb{R}))$. 
Example 5.1 Consider $f(x)=\frac{1}{\sqrt{2 \pi}} e^{-\frac{x^{2}}{2}}, x \in \mathbb{R}, u_{0}(x)=\cos x, x \in \mathbb{R}$ and $\nu=f \lambda$ where $\lambda$ is the Lebesgue measure on $\mathbb{R}$. It is obvious that $\nu \in m_{F}(\mathbb{R})$ and $u_{0} \in A P(\mathbb{R})$. With these notations (4.11) becomes

$$
\left\{\begin{array}{l}
\frac{d u(t)}{d t}(x)=\int_{\mathbb{R}} u(t)(x-y) \frac{1}{\sqrt{2 \pi}} e^{-\frac{y^{2}}{2}} d y, \quad x, t \in \mathbb{R}, \\
u(0)(x)=\cos x, \quad x \in \mathbb{R}
\end{array}\right.
$$

where $u \in \mathcal{C}^{1}(\mathbb{R}, A P(\mathbb{R}))$. For all $t \in \mathbb{R}$ we have that

$$
e^{t \nu}=\delta_{0}+t \frac{f \lambda}{1 !}+\cdots+t^{n} \frac{f^{[n]} \lambda}{n !}+\cdots,
$$

where $f^{[n]}=f * f * \cdots * f$. Some calculations give us that

$$
\begin{gathered}
f^{[n]}(x)=\frac{1}{\sqrt{2 n \pi}} e^{-\frac{x^{2}}{2 n}}, \quad x \in \mathbb{R}, \quad n \in \mathbb{N}^{*}, \\
f^{[n]} \lambda * u_{0}(x)=\frac{1}{\sqrt{2 n \pi}} \int_{\mathbb{R}} \cos (x-y) e^{-\frac{y^{2}}{2 n}} d y=e^{-\frac{n}{2}} \cos x, x \in \mathbb{R}, n \in \mathbb{N}^{*} .
\end{gathered}
$$

Finally we obtain

$$
u(t)(x)=\cos x+\sum_{n=1}^{\infty} \frac{e^{-\frac{n}{2}} t^{n}}{n !} \cos x=e^{\frac{t}{\sqrt{e}}} \cos x, \quad x, t \in \mathbb{R} .
$$

In the second Cauchy problem, the solution is a function $u \in \mathcal{C}^{1}(\mathbb{R}, a p(\mathbb{R}))$. Example 5.2 Consider $f(x)=\frac{1}{\sqrt{2 \pi}} e^{-\frac{x^{2}}{2}}, x \in \mathbb{R}, \quad u_{0}=\cos (\cdot) \lambda \quad$ and $\nu=f \lambda$, where $\lambda$ is the Lebesgue measure on $\mathbb{R}$. It is obvious that $\nu \in m_{F}(\mathbb{R})$ and $u_{0} \in a p(\mathbb{R})$. With these notations (4.10) becomes

$$
\left\{\begin{array}{l}
\frac{d u(t)}{d t}(\varphi)=\int_{\mathbb{R}}\left(\int_{\mathbb{R}} \varphi(x+y) d[u(t)](x)\right) \frac{1}{\sqrt{2 \pi}} e^{-\frac{y^{2}}{2}} d y, \quad t \in \mathbb{R}, \\
u(0)=\cos (\cdot) \lambda
\end{array}\right.
$$

where $\varphi \in K(\mathbb{R})$ and $u \in \mathcal{C}^{1}(\mathbb{R}, a p(\mathbb{R}))$. In a similar manner by that used in Example 5.1 we obtain that

$$
u(t)=\left[e^{\frac{t}{\sqrt{e}}} \cos (\cdot)\right] \lambda, \quad t \in \mathbb{R} .
$$

Finally, we establish equalities for the mean of some classes of almost periodic measures depending on the parameter $t \in \mathbb{R}$. Let $u \in \mathcal{C}^{1}(\mathbb{R}, a p(G))$. It is easy to see that the function $t \in \mathbb{R} \rightarrow M[u(t)] \in \mathbb{C}$ satisfies the equality

$$
\frac{d}{d t} M[u(t)]=M\left[\frac{d u}{d t}(t)\right], \quad t \in \mathbb{R} .
$$

Next, we apply (5.14) to the solutions of the Cauchy problems (4.10) and (4.12). Consider $\nu \in m_{F}(G), \mu \in a p(G)$ such that $M(\mu) \neq 0$ and $g \in A P(G)$. First, from (5.14) we deduce the equality

$$
\frac{d}{d t} M\left[e^{t \nu} * \mu\right]=[\nu(G)] M\left[e^{t \nu} * \mu\right], \quad t \in \mathbb{R} .
$$


We use a property of the mean, respectively, the equality

$$
M\left(e^{t \nu} * \mu\right)=e^{t \nu}(G) M(\mu), \quad t \in \mathbb{R},
$$

and we obtain that the following equality holds true:

$$
\frac{d}{d t}\left[e^{t \nu}(G)\right]=\nu(G) e^{t \nu}(G), \quad t \in \mathbb{R} .
$$

On the other hand, from (5.14), it also follows that

$$
\frac{d}{d t} M\left[e^{t g} \mu\right]=M\left[g e^{t g} \mu\right], \quad t \in \mathbb{R} .
$$

In the same manner we obtain that for all $n \in \mathbb{N}^{*}$ we have,

$$
\begin{aligned}
& \frac{d^{n}}{d t^{n}} M\left[e^{t \nu} * \mu\right]=[\nu(G)]^{n} M\left[e^{t \nu} * \mu\right], \quad t \in \mathbb{R}, \\
& \frac{d^{n}}{d t^{n}}\left[e^{t \nu}(G)\right]=[\nu(G)]^{n}\left[e^{t \nu}(G)\right], \quad t \in \mathbb{R} .
\end{aligned}
$$

\section{References}

[1] L. N. Argabright and J. G. Lamadrid, Fourier Analysis of Unbounded Measures on Locally Compact Abelian Groups, Mem. Amer. Math. Soc. No. 145, 1974.

[2] L. N. Argabright and J. G. Lamadrid, Almost Periodic Measures, Mem. Amer. Math. Soc. No. 428, 1990.

[3] V. Barbu, Nonlinear Semigroups and Differential Equations in Banach Spaces, Editura Academiei, Bucureşti, 1976.

[4] N. Dinculeanu, Vector Measures, Veb Deutscher Verlag Der Wissenschaften, Berlin, 1966.

[5] N. Dinculeanu, Integrarea pe Spatii Local Compacte, (in romanian), Editura Academiei R.P.R., Bucureşti, 1965.

[6] J. A. Goldstein, Semigroups of Linear Operators and Applications, Oxford University Press, New York, 1985.

[7] E. Hewitt and K. A. Ross, Abstract Harmonic Analysis. Vol. I. Springer - Verlag, Berlin, Göttingen, Heidelberg, 1963.

[8] E. Hille and R. S. Phillips, Functional Analysis and Semi - Groups, American Mathematical Society, Providence, 1957.

[9] J. G. Lamadrid, Sur les Mesures Presque Périodiques, Séminaire KGB sur les marches aléatoires, Astérisque 4, 1973.

[10] W. Rudin, Fourier Analysis on Groups, Interscience Tracts in Pure and Applied Mathematics, Number 12, Interscience Publishers - John Wiley and Sons, New York, London, 1962.

[11] T. Vladislav and I. Raşa, Analiză Numerică. Aproximare, Problema lui Cauchy Abstractă, Proiectori Altomare, (in romanian), Editura Tehnică, Bucureşti, 1999. 